\title{
Optimizing access to matched therapies
}

the traditional
trial design
paradigm
for precision
oncology trials
needs to be
revised

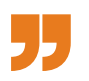

Oncology precision medicine trials typically have low rates of matching molecular aberrations with rational agents. Now, three precision medicine trials have used strategies that can improve matching rates.

The I-PREDICT trial involved patients with previously treated advanced-stage solid tumours: 73 patients received at least one molecularly matched agent (a gene product-targeted agent, an immunecheckpoint inhibitor, a hormonal therapy or a cytotoxic agent) and 10 patients received standard-of-care treatments (owing to clinician or patient preferences, lack of clinical trial availability or risk of toxicities).

A matching score (MS) was calculated for each patient. Median progression-free survival (PFS) and overall survival (OS) durations were longer in patients with a high MS $(>50 \%)$ than in those with a low MS ( $\leq 50 \%)$ : 6.5 months versus 3.1 months $(P=0.001)$ and not reached versus 10.2 months $(P=0.046)$, respectively.

The ratio of PFS reported in the trial (PFS2) to PFS on the therapy received immediately before enrolment (PFS1) was calculated. More patients with a high MS had a PFS ratio $\geq 1.3$ : $75 \%$ versus $36.6 \%$ $(P=0.026)$. The incidence of grade $\geq 3$ treatment-related adverse events

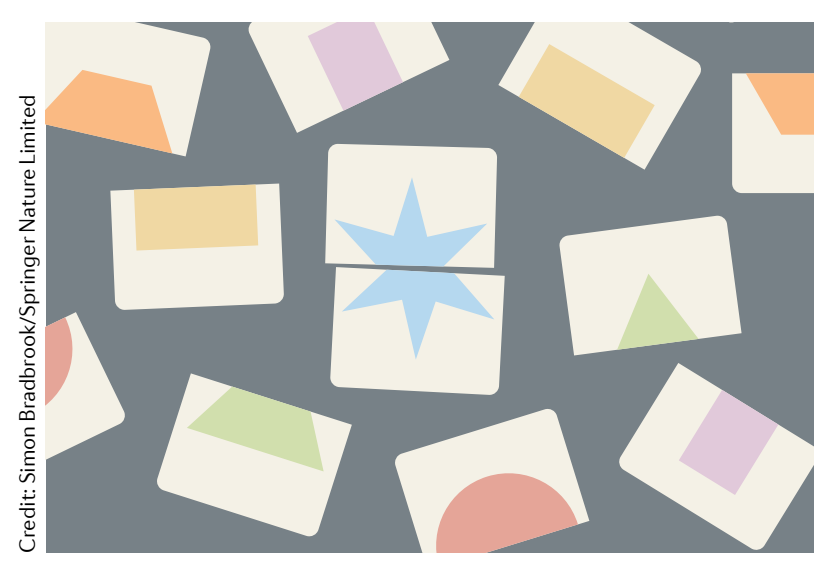

(TRAEs) was $20 \%, 15.6 \%$ and $3.6 \%$ in patients receiving a non-matched treatment, treatment with a low MS and treatment with a high MS, respectively.

The matching rate in I-PREDICT was $49 \%$. "For a precision medicine approach to work, we need to impact multiple targets. Our findings provide evidence that the traditional trial design paradigm for precision oncology trials needs to be revised," summarizes investigator Jason Sicklick.

In the WINTHER trial, patients with previously treated advanced-stage solid tumours received a treatment chosen according to the results of DNA profiling (arm A; $n=69$ ) or RNA profiling ( $\operatorname{arm~B;} n=38) .63$ patients received monotherapies and 44 patients received combinations. Similar to I-PREDICT, a broad range of therapeutic agents was administered. "In this trial, the matching rate was $35 \%$. Without transcriptomic data, the matching rate would have been $23 \%$," comments investigator Razelle Kurzrock.

The primary objective of WINTHER, a PFS2:PFS1 ratio $>1.5$ in $50 \%$ of patients in arm $\mathrm{A}$ and in $40 \%$ in $\operatorname{arm~B,~was~not~achieved~}(20.3 \%$ and $26.3 \%$, respectively). Median OS durations were 5.1 months and 7.4 months, respectively. An ECOG performance status of 0 (versus $>0$ ) and a high MS (versus a low MS) were associated with longer OS (25.8 months versus 4.5 months; $P<0.0001)$. Grade $\geq 3$ TRAEs were reported in none of the patients with a low MS and in $17 \%$ with a high MS.

In both I-PREDICT and WINTHER, biopsy samples were the main source of DNA used to inform treatment decisions. Circulating tumour DNA (ctDNA) can also provide valuable information. "The aim of Part A of the TARGET trial was to demonstrate the feasibility of testing for genomic alterations in ctDNA and turn around the results in a meaningful timeframe to influence clinical decision making," explains investigator Matthew Krebs.

Part A of TARGET involved 100 patients with advanced-stage solid tumours (22 different types). Sequencing data were obtained from ctDNA in $99 \%$ of the patients and from tumour tissue in $95 \%$. For the overlapping genes, a 78.3\% level of concordance was observed between analyses of ctDNA and those of tumour tissue.

"Our ctDNA assay includes 641 genes and takes into account the broad range of tumour types seen in the phase I setting," comments investigator Caroline Dive. 70 patients had $\geq 1$ mutation detected; 41 patients had an alteration considered to be actionable, 11 of whom were subsequently assigned to receive a matched therapy in a clinical trial. The median duration of treatment was 6 months and the response rate was $36 \%$ for patients receiving a matched therapy. "Our data support the use of both tumour and ctDNA analysis to maximize the chance of finding actionable alterations," concludes Krebs.

The teams involved in these trials are now conducting various follow-up trials. If high matching rates are consistently achieved in future studies, precision oncology approaches will become increasingly feasible.

Diana Romero

ORIGINAL ARTICLES Sicklick, J. K. et al. Molecular profiling of cancer patients enables personalized combination therapy: the I-PREDICT study. Nat. Med. https://doi.org/10.1038/s41591019-0407-5 (2019) |Rodon, J. et al. Genomic and transcriptomic profiling expands precision cancer medicine: the WINTHER trial. Nat. Med. https:// doi.org/10.1038/s41591-019-0424-4 (2019) | Rothwell, D. G. et al. Utility of ctDNA to support patient selection for early phase clinical trials: the TARGET study. Nat. Med. https://doi.org/10.1038/ s41591-019-0380-z (2019) 